\title{
On the Finding the Other Eigenvalues and Eigen Functions and Ortogonal Basis with a Nonlocal Parity Condition of the Third Kind
}

\author{
Naser Abbasi ${ }^{1}$,, Hamid Mottaghi Golshan ${ }^{2}$, Mahmood Shakori ${ }^{1}$ \\ ${ }^{1}$ Department of Mathematics, Lorestan University, Khoramabad, Iran \\ ${ }^{2}$ Department of Mathematics, Islamic Azad University, Ashtian Branch, Ashtian, Iran
}

Email address:

Naserabbasi_persia@yahoo.com (N. Abbasi),motgolham@gmail.com (H. M. Golshan), mahmoodshakoori@gmail.com (M. Shakori)

To cite this article:

Naser Abbasi, Hamid Mottaghi Golshan, Mahmood Shakori. On the Finding the Other Eigenvalues and Eigen Functions and Ortogonal Basis with a Nonlocal Parity Condition of the Third Kind. Pure and Applied Mathematics Journal. Vol. 4, No. 6, 2015, pp. 259-263.

doi: 10.11648/j.pamj.20150406.16

\begin{abstract}
In the present paper, we find out the eigenvalues and the corresponding eigenfunctions of the modified Frankl problem with a nonlocal parity condition, the completeness and the basis property in the elliptic part of the third kind of a domain in $L^{2}\left(0, \frac{\pi}{2}\right)$. We also consider a new boundaries condition and analyze the orthogonal basis of the eigenfunctions depending on parameters of the problem.
\end{abstract}

Keywords: Frankl Problem, Lebesgue Integral, Holder Inequality, Bessel Equation

\section{Introduction}

The classical Frankl problem was considered in [3]. The problem was further developed in [2, pp.339-345], [12, pp.235-252]. Several authors have also have investigated this issue (see [1-13]). On the solution of the Frankl prolem in a special domain in [12]. About spectrum of the gasedynamic problem of Frankl for the model equation of mixed type in [10]. About construction of the gasedynamic problem of Frankl in [11]. Basis property of eigen -functions of the generalized problem of Frankl with a nonlocal parity condition and with the discontinuity of the gradient of solution in [9].

In the present paper, we consider boundaries conditions of the third kind on the intervals $(-1,0)$ and $(0,1)$ of the axis OY for which the derivatives of functions with respect to $x$ on these intervals are related by linear dependence. We show that if the dependence coefficient exceeds -1 (the coefficient cannot be zero, since, otherwise, the problem will degenerate), then the systems of eigenfunctions of the problem forms a Riesz basis in the elliptic part of the domain.

\section{Statement of the Modified Frankl Problem}

Definition 1. Find a solution

$$
u(x, y) \in C^{0}\left(\overline{D_{+} \cup D_{-1} \cup D_{-2}}\right) \cap C^{2}\left(D_{-1}\right) \cap C^{2}\left(D_{-2}\right),
$$

of the modified generalized Frankl problem

$$
u_{x x}+\operatorname{sgn}(y) u_{y y}+\mu^{2} \operatorname{sgn}(x+y) u=0 .
$$

in $D_{+} \cup D_{-}$and the boundary conditions

$$
u(1, \theta)=0, \theta \in\left[0, \frac{\pi}{2}\right],
$$

in the polar coordinate system

$$
u(0, y)=0, y \in[-1,1]
$$

$k \frac{\partial u}{\partial x}(0, y)=\frac{\partial u}{\partial x}(0,-y), y \in(0,1), k \neq 0$ is a constant 


$$
k \lim _{y \rightarrow+0} u(x, y)=\lim _{y \rightarrow-0} u(x, y), x \in(0,1) .
$$

here $D_{+}$is the domain in the top half-plane bounded by a circle

$$
\gamma=\left\{(x, y), x^{2}+y^{2}=1\right\}
$$

and the segment $[0,1]$ of the axis OY, and $D_{-}=D_{-1} \cup D_{-2}$ is the domain in the bottom half space, where $D_{-1}$ is bounded by the characteristic $y=x-1$ and $y=-x$ and the segment $[0,1]$ of the axis OX and $D_{-2}$ is bounded by the characteristic $y=x-1$ and $y=-x$ and the segment $[-1,0]$ of the axis OY.

Definition 2. .System $\left\{x_{n}\right\}_{n \in N} \subset X$ is called complete in $X$ if $\overline{L\left[\left\{x_{n}\right\}_{n \in N}\right]}=X$.

Definition 3. . System $\left\{x_{n}\right\}_{n \in N} \subset X$ is called minimal in $X$ if $x_{k} \notin \overline{L\left[\left\{x_{n}\right\}_{n \in N}\right]}, \forall k \in N$.

Remark 4. If the system is minimal in $L_{p}(I)$, then it is also minimal in $L_{p}(J)$, for $J \supset I$, and if it is complete in $L_{p}(J)$ for $J \subset I$.

Theorem 5 ([1,5]). The eigenvalues and eigenfunctions of problem (1-5) can be written out in two series.

In the first series, the eigenvalues $\lambda=\mu_{n k}^{2}$ are found from the equation

$$
J_{4 n}\left(\mu_{n k}\right)=0
$$

where $\mu_{n k}, n, k=1,2, \ldots$, are roots of the Bessel equation $(6), J_{\alpha}(z)$, is the Bessel function [6,7, Russian translation], and the eigenfunctions are given by the formulas

$$
\begin{aligned}
& \tilde{u}_{n k}(r, \theta)=A_{n k} J_{4 n}\left(\mu_{n k} r\right) \sin \left(4 n\left(\frac{\pi}{2}-\theta\right)\right), \text { in } D_{+1}, \\
& \tilde{u}_{n k}(r, \theta)=k A_{n k} J_{4 n}\left(\mu_{n k} \rho\right) \sinh (4 n \psi), \quad \text { in } D_{-1}, \\
& \tilde{u}_{n k}(r, \theta)=k A_{n k} J_{4 n}\left(\mu_{n k} R\right) \sinh (4 n \varphi), \quad \text { in } D_{-2},
\end{aligned}
$$

where

$$
\begin{aligned}
& x=r \cos \theta, y=r \sin \theta \quad 0 \leq \theta \leq \frac{\pi}{2}, r^{2}=x^{2}+y^{2} \text { in } D_{+}, \\
& 0<\rho<1,-\infty<\psi<0, \rho^{2}=x^{2}-y^{2}, \quad \text { in } D_{-1}, \quad \text { and, }
\end{aligned}
$$
$x=R \sinh \varphi, y=-R \cosh \varphi, \quad 0<\varphi<+\infty, R^{2}=y^{2}-x^{2}$ in $D_{-2}$.

In the second series, the eigenvalues $\tilde{\lambda}=\tilde{\mu}_{n k}^{2}$ are found from the equation

$$
J_{\tilde{\alpha}_{n}}\left(\tilde{\mu}_{n k}\right)=0
$$

where $\Delta=\frac{1}{\pi} \arcsin \frac{\kappa}{\sqrt{1+\kappa^{2}}}, \Delta \in\left(-\frac{1}{2}, \frac{1}{2}\right) \backslash\{0\}$ $\tilde{\alpha}_{n}=2+4(n-\Delta)\left\{x_{n}\right\}_{n \in N} \subset X \quad$ and the corresponding eigenfunctions have the form

$$
\begin{aligned}
\tilde{u}_{n k}(r, \theta) & =\widetilde{A}_{n k} J_{\alpha_{n}}\left(\tilde{\mu}_{n k} r\right) \sin \left(\widetilde{\alpha_{n}}\left(\frac{\pi}{2}-\theta\right)\right), \text { in } D_{+1}, \\
\tilde{u}_{n k}(r, \theta) & =k \widetilde{A}_{n k} J_{\widetilde{\alpha}_{n}}\left(\tilde{\mu}_{n k} \rho\right) \sinh \left(\frac{2 x}{x^{2}+1} \cosh \left(\widetilde{\alpha_{n}} \psi\right)\right. \\
& \left.-x \frac{x^{2}-1}{\tilde{x}^{2}+1} \sinh \left(\widetilde{\alpha_{n}} \psi\right)\right), \quad \text { in } D_{-1}, \\
\tilde{u}_{n k}(r, \theta) & =k \widetilde{A}_{n k} J_{\widetilde{\alpha}_{n}}\left(\tilde{\mu}_{n k} R\right) \sinh \left(\widetilde{\alpha_{n}} \varphi\right), \quad \text { in } D_{-2} .
\end{aligned}
$$

Theorem 6 ([5]). The function system

$$
\left\{\cos (4 n)\left(\frac{\pi}{2}-\theta\right)\right\}_{n=0}^{\infty},\left\{\cos 4(n+\triangle)\left(\frac{\pi}{2}-\theta\right)\right\}_{n=1}^{\infty}
$$

is complete and a Riesz basis in $L^{2}\left(0, \frac{\pi}{2}\right)$, provided that $\Delta \in\left(\frac{-1}{4}, \frac{1}{2}\right) \cup\left(\frac{1}{2}, \frac{3}{4}\right)$.

\section{Main Results}

Consider Frankl problem (1)-(5) with the new boundary condition

$$
\frac{\partial u}{\partial x}(0, y)=0, y \in(-1,0) \cup(0,1)
$$

$$
k u(0, y)=u(0,-y), y \in[0,1], k u\left(0,0^{+}\right)=u\left(0,0^{-}\right),
$$

$k \frac{\partial u}{\partial y}\left(x, 0^{+}\right)=\frac{\partial u}{\partial y}\left(x, 0^{-}\right),-\infty<k<+\infty, 0<x<1$.

Theorem 7. The eigenvalues and eigenfunctions of problem (1-5) can be written out in two series.

In the first series, the eigenvalues $\lambda=\mu_{n k}^{2}$ are found from the equation

$$
J_{4 n+\Delta}\left(\mu_{n k}\right)=0
$$

where $\mu_{n k}, n, k=1,2, \ldots$, are roots of the Bessel equation (6), $J_{\alpha}(z)$, is the Bessel function [4], and the eigenfunctions are given by the formula

$$
u_{n k}=\left\{\begin{array}{cc}
A_{n k} J_{4 n}\left(\mu_{n k} r\right) \cos (4 n)\left(\frac{\pi}{2}-\theta\right), & \text { in } D^{+} ; \\
k A_{n k} J_{4 n}\left(\mu_{n k} \rho\right) \cosh (4 n) \psi, & \text { in } D_{-1} ; \\
k A_{n k} J_{4 n}\left(\mu_{n k} R\right) \cosh (4 n) \varphi, & \text { in } D_{-2},
\end{array}\right.
$$


where

$x=r \cos \theta, y=r \sin \theta$,for $0 \leq \theta \leq \frac{\pi}{2}, r^{2}=x^{2}+y^{2}$ in $D_{+} \quad, \quad x=\rho \cosh \psi, y=\rho \sinh \psi, \quad$ for, $0<\rho<1,-\infty<\psi<0, \rho^{2}=x^{2}-y^{2}, \quad$ in $D_{-1}, \quad$ and, $x=R \sinh \varphi, y=-R \cosh \varphi$, for, $0<\varphi<+\infty, R^{2}=y^{2}-x^{2}$ in $D_{-2}$.

In the second series, the eigenvalues $\tilde{\lambda}=\tilde{\mu}_{n k}^{2}$ are found from the equation

$$
J_{4(n+\Delta)}\left(\tilde{\mu}_{n k}\right)=0
$$

where $n, k=1,2, \ldots$ and the $\left(\tilde{\mu}_{n k}\right)$ are the roots of the Bessel equation (8).

$$
u_{n k}=\left\{\begin{array}{c}
\tilde{A}_{n k} J_{4(n+\Delta)}\left(\tilde{\mu}_{n k} r\right) \cos 4(n+\Delta)\left(\frac{\pi}{2}-\theta\right), \quad \text { in } D^{+} ; \\
\tilde{A}_{n k} J_{4(n+\Delta)}\left(\tilde{\mu}_{n k} \rho\right) \cdot \\
{\left[\cosh 4(n+\triangle) \varphi \cos 4(n+\triangle) \frac{\pi}{2} \quad \text { in } \quad D_{-1} ;\right.} \\
+\kappa \sinh 4(n+\triangle) \psi \cos 4(n+\triangle)], \\
k \tilde{A}_{n k} J_{4(n+\Delta)}\left(\tilde{\mu}_{n k} R\right) \cdot \\
\cosh (4(n+\triangle) \varphi) \cdot \\
{\left[\cos 4(n+\triangle) \frac{\pi}{2}-\sin 4(n+\triangle) \frac{\pi}{2}\right],}
\end{array}\right.
$$

where $\Delta=\frac{1}{\pi} \arcsin \frac{\kappa}{\sqrt{1+\kappa^{2}}}, \Delta \in\left(0, \frac{1}{2}\right)$, and

$$
\begin{gathered}
A_{n k}^{2} \int_{0}^{1} J_{4 n}^{2}\left(\mu_{n k} r\right) r d r=1, \\
\tilde{A}_{n k}^{2} \int_{0}^{1} J_{4 n+\Delta}^{2}\left(\tilde{\mu}_{n k} r\right) r d r=1, \\
A_{n k}>0 \text { and } \tilde{A}_{n k}>0 .
\end{gathered}
$$

Theorem 8 . The function system

$$
\left\{\cos (4 n)\left(\frac{\pi}{2}-\theta\right)\right\}_{n=0}^{\infty},\left\{\cos 4(n+\Delta)\left(\frac{\pi}{2}-\theta\right)\right\}_{n=1}^{\infty}
$$

is complete and a Riesz basis in $L^{2}\left(0, \frac{\pi}{2}\right)$, provided that $\Delta \in\left(\frac{-1}{4}, \frac{1}{2}\right)$

Proof. In order to prove this theorem we use the method in $[1,6]$ by considering convergence function

$$
f(\theta)=\sum_{n=0}^{\infty} A_{n} \cos 4 n\left(\frac{\pi}{2}-\theta\right)+\sum_{n=1}^{\infty} B_{n} \cos 4(n+\triangle)\left(\frac{\pi}{2}-\theta\right)
$$

in $L^{2}\left(0, \frac{\pi}{2}\right)$ and Riesz basis the system $\left(\sin 4(n+\triangle)\left(\frac{\pi}{2}-\theta\right)\right)$ for $\Delta \in\left(\frac{-1}{4}, \frac{3}{4}\right)$.

Remark 9. For $\Delta<\frac{-1}{4}$ the system (10) is not complete but is minimal, for $\Delta>\frac{3}{4}$ is complete but is not minimal, and if $\Delta=\frac{-1}{4}$, is complete and minimal.

Theorem 10. The system of eigenfunctions

$$
\begin{aligned}
& u_{n k}(r, \theta)=A_{n k} J_{4 n}\left(\mu_{n k} r\right) \cos (4 n)\left(\frac{\pi}{2}-\theta\right), \\
& \tilde{u}_{n k}(r, \theta)=\tilde{A}_{n k} J_{4(n+\Delta)}\left(\tilde{\mu}_{n k} r\right)\left[\cosh 4(n+\Delta) \varphi \cos 4(n+\Delta) \frac{\pi}{2}\right], \\
& \text { is complete and basis in the space } L^{2}\left(0, \frac{\pi}{2}\right) \text {, therefore } \\
& \qquad \int_{0}^{\frac{\pi}{2}} f(r, \theta) u_{n k}(r, \theta) r d r d \theta=0, \\
& \int_{0}^{\frac{\pi}{2}} f(r, \theta) \tilde{u}_{n k}(r, \theta) r d r d \theta=0, \\
& \text { and } f \in L^{2}\left(0, \frac{\pi}{2}\right) \text { then } f=0 \text { in }\left(0, \frac{\pi}{2}\right) .
\end{aligned}
$$

Proof. Using Fubini theorem for any $n, k=1,2, \ldots$ we have

$$
\begin{gathered}
0=\int_{0}^{\frac{\pi}{2}} f(r, \theta) u_{n k}(r, \theta) r d \theta d r \\
\int_{0}^{1}\left(r J_{4 n}\left(\mu_{n k} r\right) \int_{0}^{\frac{\pi}{2}} f(r, \theta) \cos (4 n)\left(\frac{\pi}{2}-\theta\right) d \theta\right) d r
\end{gathered}
$$

again since $f \in L^{2}\left(0, \frac{\pi}{2}\right)$ so;

$$
\int_{0}^{1} \int_{0}^{\frac{\pi}{2}}|f(r, \theta)|^{2} d \theta d r<\infty
$$

and since the system $\left\{\sqrt{r} J_{4 n}\left(\mu_{n k} r\right)\right\}_{k=1}^{\infty}$ in $L^{2}(0,1)$ is orthogonal and complete, it is enough to prove:

$$
\sqrt{r} \int_{0}^{\frac{\pi}{2}} f(r, \theta) \cos (4 n)\left(\frac{\pi}{2}-\theta\right) d \theta \in L^{2}(0,1) .
$$


Using the Holder inequality

$$
\begin{gathered}
\left|\sqrt{r} \int_{0}^{\frac{\pi}{2}} f(r, \theta) \cos (4 n)\left(\frac{\pi}{2}-\theta\right) d \theta\right|^{2}<\frac{1}{2} r \int_{0}^{\frac{\pi}{2}}\left|f^{2}(r, \theta)\right| d \theta \int_{0}^{\frac{\pi}{2}} d \theta \\
=\frac{\pi}{4} r \int_{0}^{\frac{\pi}{2}}|f(r, \theta)|^{2} d \theta=\frac{\pi}{4} r \int_{0}^{\frac{\pi}{2}}|f(r, \theta)|^{2} d \theta,
\end{gathered}
$$

with the integration interval $(0,1)$.

$$
\begin{aligned}
& \int_{0}^{1} \mid \sqrt{r} \int_{0}^{\frac{\pi}{2}}\left.f(r, \theta) \cos (4 n)\left(\frac{\pi}{2}-\theta\right) d \theta\right|^{2} d r ? \\
&<\frac{\pi}{4} \int_{0}^{1} \int_{0}^{\frac{\pi}{2}} r|f(r, \theta)|^{2} d r d \theta<\infty
\end{aligned}
$$

This inequality is equivalent to

$$
\left\{\int_{0}^{1} \sqrt{r}\left|\int_{0}^{\frac{\pi}{2}} f(r, \theta) \cos (4 n)\left(\frac{\pi}{2}-\theta\right) d \theta\right|^{2} d r\right\}^{\frac{1}{2}}<\infty .
$$

Also system $\left\{\sqrt{r} J_{4 n}\left(\mu_{n k} r\right)\right\}_{k=1}^{\infty}$ is orthogonal and complete in $L^{2}\left(0, \frac{\pi}{2}\right)$ of relation

$$
\int_{0}^{1}\left(\sqrt{r} J_{4 n}\left(\mu_{n k} r\right) \sqrt{r} \int_{0}^{\frac{\pi}{2}} f(r, \theta) \cos (4 n)\left(\frac{\pi}{2}-\theta\right) d \theta\right) d r=0
$$

imply that

$$
\sqrt{r} \int_{0}^{\frac{\pi}{2}} f(r, \theta) \cos (4 n)\left(\frac{\pi}{2}-\theta\right) d \theta=0
$$

According to theorem 6, we conclude that $f(r, \theta)=0$ in $L^{2}(0,1)$. Similarly, if we consider the above calculations for sequence $\left\{\cos 4(n+\triangle)\left(\frac{\pi}{2}-\theta\right)\right\}_{n=1}^{\infty}$, we have

$$
\sqrt{r} \int_{0}^{\frac{\pi}{2}} f(r, \theta) \cos 4(n+\triangle)\left(\frac{\pi}{2}-\theta\right) d \theta=0
$$

Because completeness $\left\{\cos 4(n+\triangle)\left(\frac{\pi}{2}-\theta\right)\right\}_{n=0}^{\infty},\{r, \theta)=0$ in $L^{2}(0,1)$.

The proof of the theorem is complete.

Remark 11. If $\Delta=0$ then the system becomes the system $\{\sin (2 n \theta)\}_{n=1}^{\infty}$ which is basis in the space $L^{p}\left(0, \frac{\pi}{2}\right)$ and an orthogonal basis in the space $L^{2}\left(0, \frac{\pi}{2}\right)$.

The proof of remark 11 results from theorem 10 .

Remark 12. In case $\Delta>\frac{3}{4}$ and $\Delta \neq \frac{1}{2}+k, k \in \mathbb{N}$ then the system is complete but is not minimal.
In case $\Delta<\frac{-1}{4}$ and $\Delta \neq-\frac{1}{4}-k, k \in \mathbb{N}$ then the system is not complete but is minimal.

In case $\Delta=\frac{1}{2}+k, k \in \mathbb{Z}$ then the system is complete but is not minimal in the space $L^{2}\left(0, \frac{\pi}{4}\right)$ and is not complete in the space $L^{2}\left(0, \frac{\pi}{2}\right)$.

The proof of remark 12 results from theorem 8 .

\section{Conclusion}

Consider Frankl problem (1)-(5) with the new boundary condition (9)-(10) and (11), so we find out the eigenvalues of the problem with a nonlocal parity condition ,the completeness and the basis property in the elliptic part of the third kind of a domin in $L^{2}\left(0, \frac{\pi}{2}\right)$.

\section{Acknowledgements}

This research was partially supported by Lorestan University, Khoramabad and the authors are grateful to Academician E.I. Moiseev for his interest in this work.

\section{References}

[1] N. Abbasi, Basis property and completeness of the eigenfunctions of the Frankl problem. (Russian) Dokl. Akad.Nauk425 (2009), no. 3, 295-298; translation in Dokl. Math. 79 (2009), no. 2, 193-196.

[2] A. V. Bitsadze, Nekotoryeklassyuravneni v chastnykhproizvodnykh. (Russian) [Some classes of partial differential equations] "Nauka", Moscow, 1981. 448pp.

[3] F. Frankl, On the problems of Chaplygin for mixed sub- and supersonic flows. (Russian) Bull. Acad. Sci. URSS. Sr. Math. [Izvestia Akad. Nauk SSSR] 9, (1945).121-143.

[4] H.van Haeringen, L.P. Kok, Higher transcendental functions, Vol. II [McGraw-Hill, New York, 1953; MR 15, 419] by A. Erdlyi, W. Magnus, F. Oberhettinger and F. G. Tricomi. Math. Comp. 41 (1983), no. 164, 778.

[5] E.I. Moiseev, The basis property for systems of sines and cosines. (Russian) Dokl. Akad. Nauk SSSR 275 (1984), no.4, 794-798.

[6] N. Abbasi, E.I. Moiseev, Basis property of eigenfunctins of the Generalized Gasedynamic problem of Frankl with a nonlocal oddness conditions, integral transforms and special Functions.(England) 21 (2010), no. 4,286-294.

[7] E. I. Moiseev, N. Abbasi, The basis property of an eigenfunction of the Frankl problem with a nonlocal parity conditionin the space Sobolev $\left(\mathrm{W}_{1}^{\mathrm{p}}(0, \pi)\right)$. Integral Transforms Spec. Funct. (England) 22 (2011), no. 6, 415-421.

[8] E. I. Moiseev, On the solution of the Frankl problem in a special domain, Differ. Uravn. 8(4) (1992), pp.721-723. 
[9] E. I. Moiseev and N. Abbasi, Basis property of eigen functions of the generalized problem of Frankl with a nonlocal parity condition and with the discontinuity of the gradient of solution, Differ.Uravn.44(10)(2009),pp.155-160.

[10] K. B. Sabitov, About spectrum of the gasedynamic problem of Frankl for the model equation of mixed type, Differ. Uravn. 39(11) (2003), pp.1568-1570.

[11] K. B. Sabitov and V. V. Tikhomirov, About construction of the gasedynamic problem of Frankl, Differ.Uravn.23 (10) (1990), pp.100-109.
[12] M.M. Smirnov, Uravneniyasmeshannogotipa. (Russian) [Equations of mixed type] Izdat. "Nauka", Moscow 1970 295pp. New York, 1955.vii+329. pp.

[13] A. Zygmund, Trigonometrical series. Dover Publications, New York, 1955.vii+329. pp. 